\title{
Night Time Vehicle Detection
}

\author{
Hasan Fleyeh and Iman A. Mohammed
}

\begin{abstract}
Night driving is one of the major factors which affects traffic safety. Although detecting oncoming vehicles at night time is a challenging task, it may improve traffic safety. If the oncoming vehicle is recognised in good time, this will motivate drivers to keep their eyes on the road. The purpose of this paper is to present an approach to detect vehicles at night based on the employment of a single onboard camera. This system is based on detecting vehicle headlights by recognising their shapes via an SVM classifier which was trained for this purpose. A pairing algorithm was designed to pair vehicle headlights to ensure that the two headlights belong to the same vehicle. A multi-object tracking algorithm was invoked to track the vehicle throughout the time the vehicle is in the scene. The system was trained with 503 single objects and tested using 144587 single objects which were extracted from 1410 frames collected from 15 videos and 27 moving vehicles. It was found that the accuracy of recognition was $97.9 \%$ and the vehicle recognition rate was $96.3 \%$ which indicates clearly the high robustness attained by this system.
\end{abstract}

Keywords. Vehicle Detection, Object Tracking, SVM Shape Classification, Night Detection.

2010 Mathematics Subject Classification. 68T10, 68T45.

\section{Introduction}

Vehicle detection and tracking is an important part of driver assistance systems (DAS) which warn drivers of potential collisions. To achieve this task, robust algorithms which are able to employ robust feature extraction methods and track the targets are required. Unfortunately, these requirements are difficult to fulfil where night vehicle detection is concerned [9]. The current research indicated that much has been achieved regarding vehicle detection in the daytime. In contrast, as vehicle detection at night is a more challenging issue, it has not been researched to the same extent.

The traffic accident rate at night is higher than that during the day. Statistics show that $55 \%$ of driving fatalities occur at night while night driving represents only $25 \%$ of the whole driving period [5]. This is due to the fact that people either do not switch from high to low beam or from low to high when they should. 
A study which was carried out by the Transportation Research Institute at the University of Michigan has shown that high beam is used at only $25 \%$ when it is possible and drivers seem to forget to switch from low to high beam or are bored by incessant switch changes [16].

At night, the most reliable information about the existence of a vehicle is from its head or tail-lights, being what a driver can easily identify from other vehicles. Developing a night vehicle detection algorithm means employing these light spots as basic knowledge which can be exploited to detect and track the vehicles.

The aim of the paper is to design and implement robust night vehicle detection in complex scenes such as city streets. Such a goal is a challenge as the detection of a moving vehicle from a moving camera is difficult due to the change in the background, the presence of a huge spectrum of vehicle distribution, vehicle shape, weather conditions, and buildings by roads [9]. At night, vehicles can be recognised by the spots generated by their headlights. Categorising such spots into vehicle and non-vehicle is a classification problem which cleans the image from the irrelevant objects such as reflection from billboards or road signs [12]. Once a vehicle is detected, a tracking mechanism is then employed to follow that vehicle in the scene. This paper presents the implementation and evaluation of a night time vehicle detection algorithm.

The paper is organised as follows: A literature review is presented in Section 2. The proposed vehicle detection approach is described in Section 3 with results and discussions being presented in Section 4. Conclusions and future work can be found in Section 5.

\section{Literature Review}

In recent years, research in the area of vehicle detection both at night and during daytime has grown rapidly because of the real need for such systems in future vehicles. Performance indexes required by these systems include high recognition rates, real-time implementation, robustness for variant environments, and feasibility under poor visibility conditions.

Alcantarilla et al. [1] proposed an approach for vehicle detection in which an adaptive thresholding was invoked to segment the image taken by a black and white micro-camera. The geometric characteristics of the bright spots in the image were employed to cluster them and then the spots were tracked by Kalman filters. The tracked objects were classified into traffic signs or vehicles using a support vector machine (SVM). The proposed approach could detect vehicles within the range $300-500 \mathrm{~m}$. 
Görmer et al. [8] suggested a vehicle detection approach which was similar to the one proposed by Alcantarilla et al. [1] in the segmentation and pair selection. However, it differs in its classification part which invoked a set of four similarity features to compare the spots in the pair. These features were the covariance intersection of the headlight spots, the ratio between the spot's halo and the size of the saturated area, the vertical movement similarity of the spots, and the ratio of the deviation between the predicted neighbour spot position and the actual spot coordinates. The overall detection rate of the system was determined to be $89.3 \%$ on a frame basis, including vehicles in one or more lanes.

In the system proposed by Rebut et al. [12] local maxima were combined with region growing to segment the light spot. The SVM classifier, which achieved a $97 \%$ classification rate, was invoked to differentiate between reflections and lights, and the Kalman filter was used to track the lights. Kalman filters were collaborated with a shortest path algorithm to create the association between a blob in a certain frame and the same one in the previous frame. A cost function was used to pair the headlights of a vehicle recognised in a certain frame. The proposed approach was able to detect vehicles within the range 400-600 m.

Alt et al. [2] used a combination of hardware and software to achieve vehicle detection at night. The image segmentation and labelling was done using hardware while the rest of the processing was performed by software. The image segmentation was based on thresholding and shape filtering using different masks. Information from the tracking was used to determine static and moving lights by finding the motion vectors of the light spots. Pair finding was achieved by considering the distance between the spots, their ratio of brightness, and the existence of license plate lights between pairs. Vehicle detection with high certainty was $57 \%$, low certainty $16 \%$, and vehicle not detected $27 \%$.

Liu et al. [10] proposed a vehicle detection system based on using a single moving camera during day time. A symmetric feature selection strategy based on the Adaboost learning method was invoked for vehicle detection. Two kinds of symmetric features which are global and local were defined. They were used to construct a cascaded structure with a one-class followed by a two-class classifier to recognise vehicles from false positives. A detection rate of $99.4 \%$ was achieved by this method.

Kim et al. [9] used a fusion of sonar and vision sensors to detect and track vehicles during day and night time. The sonar sensor was used to detect the vehicle within a $10 \mathrm{~m}$ distance while images were invoked to estimate the vehicle's distance when it was $10 \mathrm{~m}$ away from the camera. For the night time condition, the vehicle features considered were headlights, taillights, brake lights and reflected lights around light sources. The performance rate of vehicle detection was $85 \%$ and $96 \%$ for vehicle tracking and restoration. 


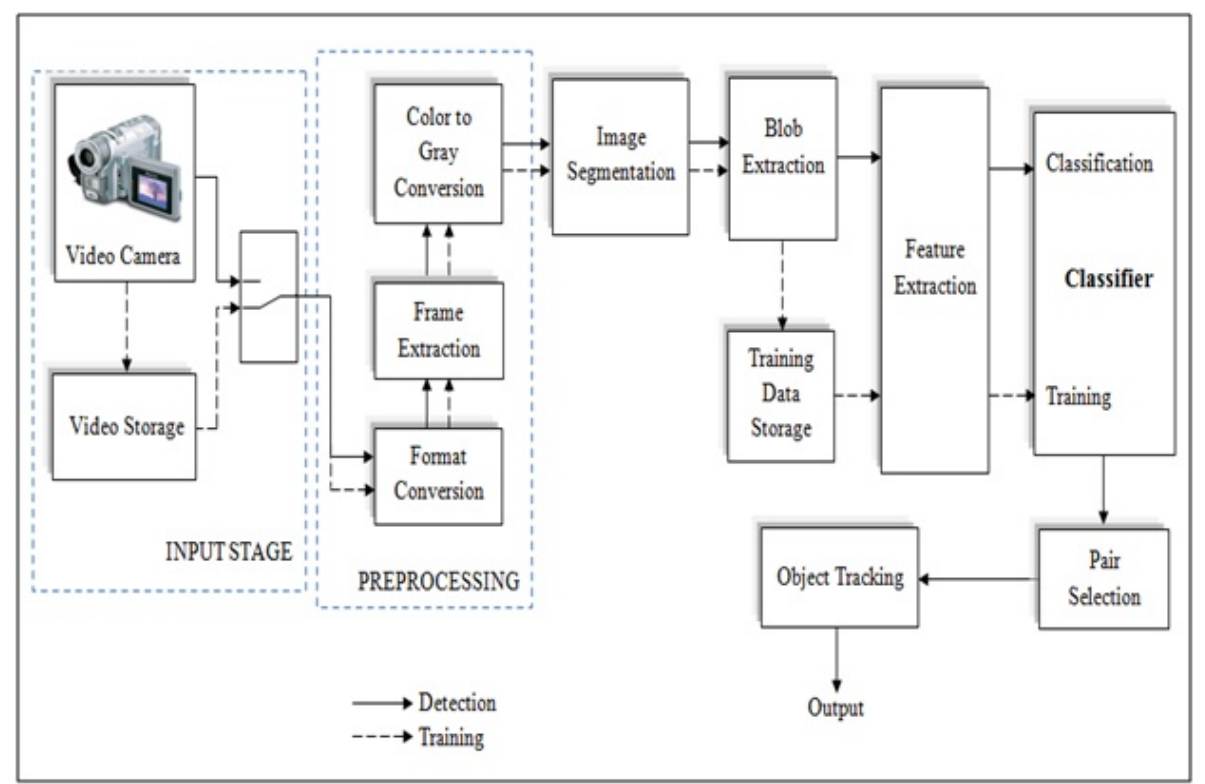

Figure 1. Block diagram of the detection algorithm.

Cucchiara and Piccardi [4] used two different approaches based on day and night time illumination conditions to detect vehicles. For the day time spatiotemporal analysis on moving templates were used while for night images morphological analysis of headlights was utilised. Once spotlights were identified, the forward chain rule base system was invoked for vehicle tracking.

\section{The Proposed Approach}

The proposed vehicle detecting algorithm has two modes: training and detection. The training mode is where some of the collected images are employed to train a classifier so that it can be used to detect headlights in the detection mode. The overall block diagram representing the algorithm including both modes and their respective modules is given in Figure 1.

\subsection{Input Stage}

Videos for training and testing were collected from a moving vehicle in central Sweden by using a Sony HDR-CX115E video camera. The camera was pointed towards the direction of the vehicle's motion from which the videos were collected. 
In total 15 videos were collected and a total of 7179 frames were produced from these videos. The videos were invoked in the training and testing of the proposed system. Objects extracted from these videos were employed to train the shape classifier. To test and validate the proposed system 1410 frames were invoked.

\subsection{Image Segmentation}

Segmentation is a crucial step in many computer vision applications. The results gained from the other stages are affected by the outcome of the segmentation stage. Therefore, choosing the right segmentation algorithm is crucial. Segmentation using the extended maxima transformation technique [14] was employed for this purpose. The $h$-value for this technique was chosen empirically. Different values of $h$ in the range $[5,100]$ were tested and the segmentation results obtained for each $h$-value are shown in Figure 2. Binary images generated by small values of $h$ were characterised by generating too many objects which are not headlights, while large values produced binary images which contains less objects but the headlights were becoming bigger and merged together, which would make detection difficult. Thus a compromise between these two extremes was made by setting the $h$-value to 15 . Figure 2 illustrates how bright spots are segmented.

The quality of segmentation was compared with two other segmentation methods, adaptive thresholding of integral images [3] and Otsu thresholding [11]. Figure 3 illustrates the results of the segmentation from these two methods compared with that produced by the extended maxima transformation method. It is obvious that the quality of segmentation produced by the extended maxima transformation is better and more suitable for the current application.

\subsection{Training Database}

The bright objects extracted from the segmented image of a road at night consist of different lights and reflections. This image may contain headlights of vehicles and non-vehicle lights such as street lights and reflection from different traffic signs, white lane marker lines and arrows on the road. Hence the objects extracting from the segmentation stage are very diverse.

The training dataset was built in such a way so as to represent these different types of objects. A total of 503 objects were extracted for training purposes. Among these objects, 158 represented vehicle lights, while the other 345 objects corresponded to lights from other sources and reflections. Table 1 illustrates a sample of these objects. 


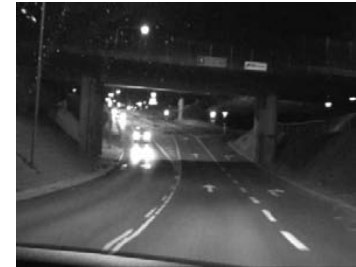

(a) Gray image

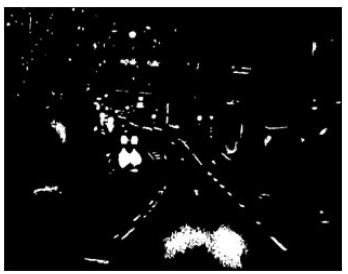

(d) $h=15$

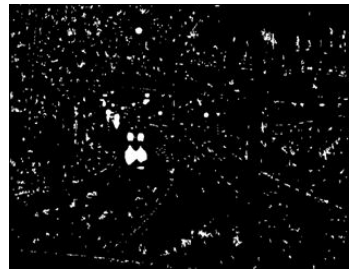

(b) $h=5$

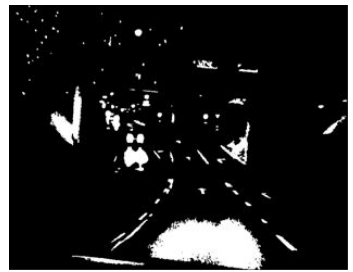

(e) $h=25$

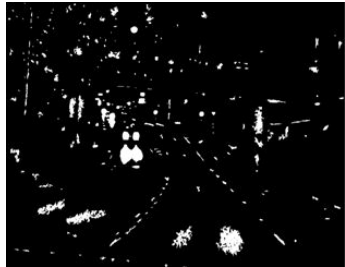

(c) $h=10$

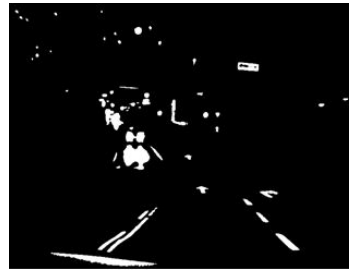

(f) $h=50$

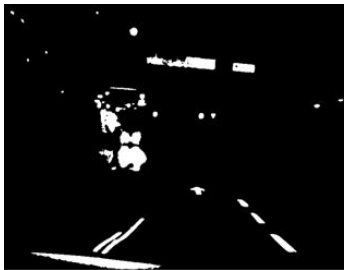

(g) $h=75$

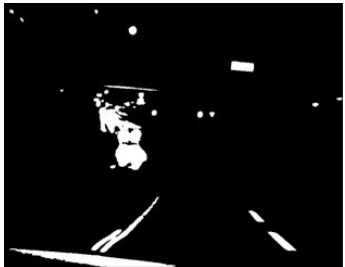

(h) $h=100$

Figure 2. Segmentation with extended maxima transformation with different values of $h$.

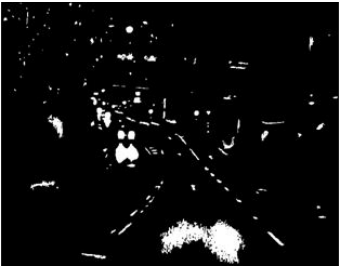

(a) Extended maxima transform

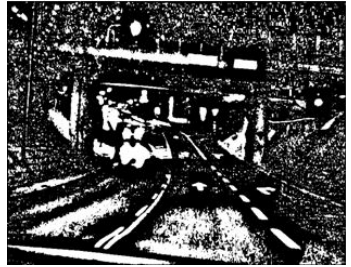

(b) Adaptive thresholding using integral image

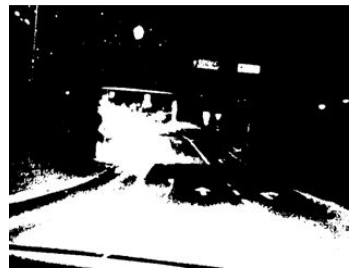

(c) Otsu thresholding

Figure 3. Comparison of different segmentation techniques. 


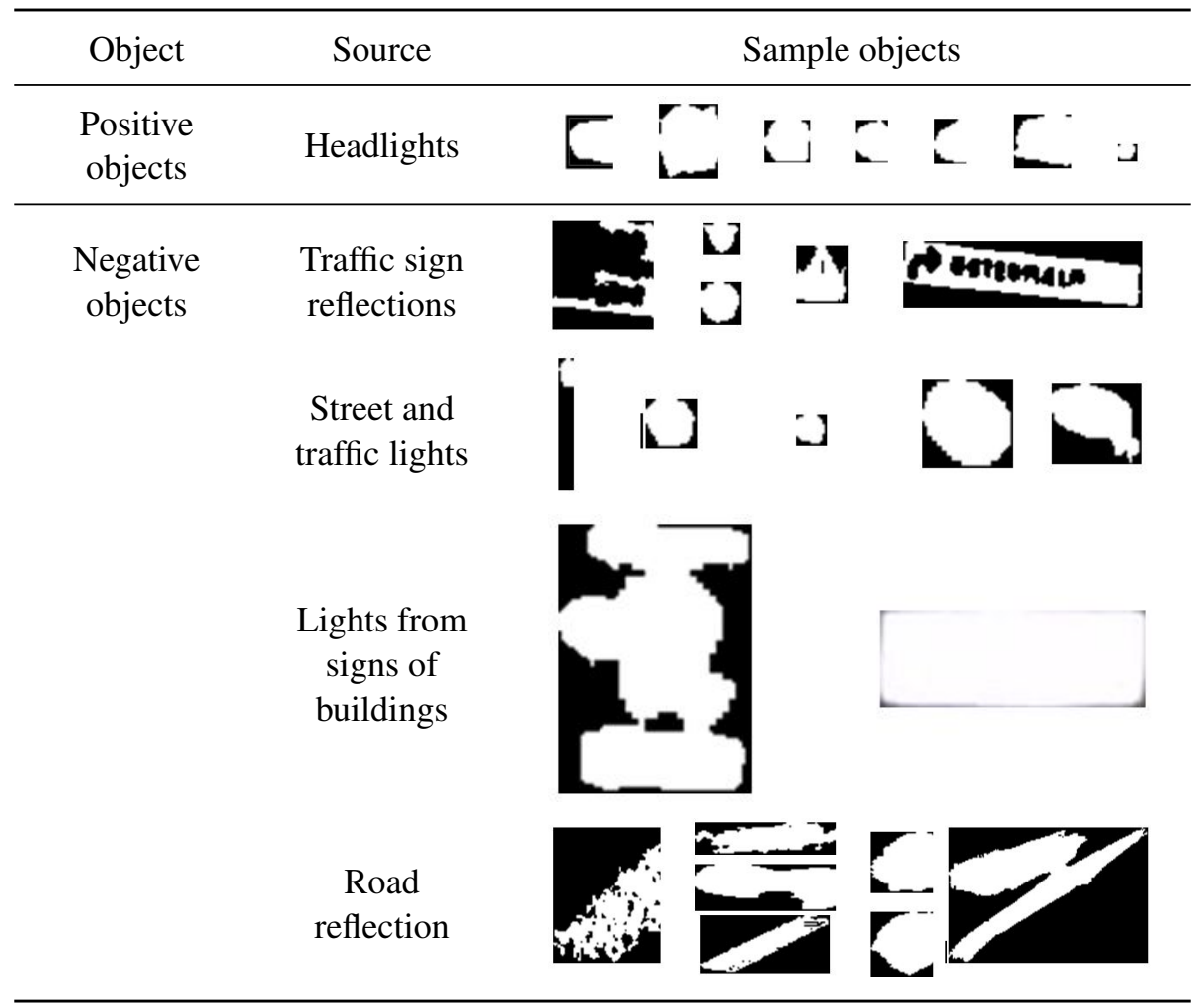

Table 1. Samples of the extracted blobs for training and testing.

\subsection{Features Extraction}

Since the characteristics of a vehicle's headlights differ from those of other objects in the scene, different types of features were considered. They can be grouped as follows:

1. Features based on shape measurements. The shapes of vehicle headlights are circular or elliptical while the other objects in the scene may be elongated, triangular or rectangular in shape. Thus the five shape measures which were considered are eccentricity, circularity, rectangularity, triangularity and ellipticity [13]. They are given in Table 2.

2. Features based on size and position. The other features considered are the size of the blob under consideration and its position. It was observed that vehicle headlights originated around the centre of the image and propagated towards its 


\begin{tabular}{|c|c|c|}
\hline Feature & Equation & Description \\
\hline Eccentricity & $\mathrm{Ecc}=\frac{\text { major axis }}{\text { minor axis }}$ & \\
\hline Circularity & $C=\frac{4 \pi A}{P^{2}}$ & $\begin{array}{l}A \text { is the area of the object and } \\
P \text { is its perimeter; } C \text { ranges } \\
0 \leq C \leq 1 \text { where } 1 \text { means a } \\
\text { perfect circle }\end{array}$ \\
\hline Rectangularity & $R=\frac{\text { region's area }}{\text { MBR area }}$ & $\begin{array}{l}\text { MBR is the minimum bounding } \\
\text { rectangle }\end{array}$ \\
\hline Triangularity & $\begin{array}{l}T= \\
\begin{cases}108 I_{1} & \text { if } I_{1} \leq \frac{1}{108} \\
\frac{1}{108 I_{1}} & \text { otherwise }\end{cases} \end{array}$ & $\begin{array}{l}T \text { ranges } 0 \leq T \leq 1 \text { where } 1 \\
\text { means a perfect triangle; } I_{1} \text { is } \\
\text { the first affine moment invariant } \\
{[6,7]}\end{array}$ \\
\hline Ellipicity & $\begin{array}{l}E= \\
\begin{cases}16 \pi^{2} I_{1} & \text { if } I_{1} \leq \frac{1}{16 \pi^{2}} \\
\frac{1}{16 \pi^{2} I_{1}} & \text { otherwise }\end{cases} \end{array}$ & $\begin{array}{l}E \text { ranges } 0 \leq E \leq 1 \text { where } 1 \\
\text { means a perfect triangle; } I_{1} \text { is } \\
\text { the first affine moment invariant } \\
{[6,7]}\end{array}$ \\
\hline
\end{tabular}

Table 2. Description of shape measures.

lower left side. On the other hand, street lights were located in the top part of the image while reflections were either to the bottom, or in other positions which were significantly different from vehicle headlights. Thus the spatial position of the extracted objects is considered as a feature which could discriminate headlights from other light sources. In addition to this, headlights have a similar size or area, while the size of other bright objects varies from very small to very large. Hence, the size of the blob can also be considered as another feature which could discriminate headlights from non-headlights. This is illustrated in Figure 4.

3. Features based on intensity. Light intensity or the gray level of the object under consideration in the gray image is another feature which can be employed to distinguish between vehicle headlights and any other objects in the scene. Vehicle headlights tended to have a higher intensity while the bright objects corresponding to non-headlights had lower intensities, as shown in Figure 5. This is due to the fact that headlights are directed in the direction of the road while the other lights are directed vertically on the road. Since the videos were collected from a camera 


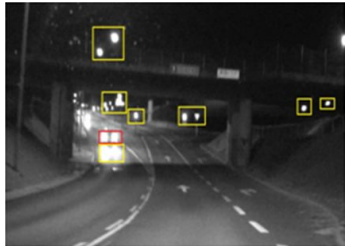

(a) Gray image

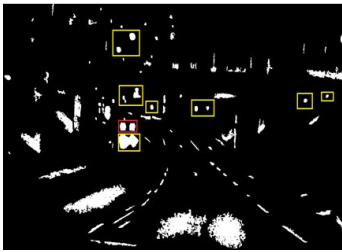

(b) Segmented image

Figure 4. Features based on size and position.
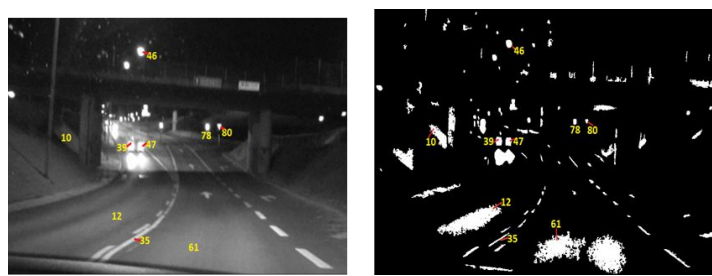

Figure 5. Features based on intensity.

which was facing the vehicle's headlights, it is obvious that the gray level of these headlights is higher than any other lights.

4. Features based on top-hat transform. Top-hat transform is a morphological operation by which small objects are extracted from the image. White top-hat transform is able to extract all objects in the image under consideration which are smaller than the structuring element and brighter than their surroundings while black top-hat transform is able to extract all objects in the image under consideration which are smaller than the structuring element and darker than their surroundings. Black top-hat transform, which is defined as the difference between the closing of an image and that image, was chosen because of its ability to detect the local valleys in light intensity between a vehicle's headlights [1]. The area invoked to quantify the local valley was specified by the size of the bounding box containing the object under consideration plus 10 pixels on each side of the bounding box. This size was chosen experimentally with the objective of capturing all the areas to detect the local valleys in the light intensity. Detected valleys between bright objects are shown in Figure 6. 


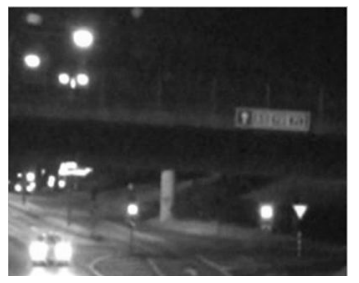

(a) Gray level image

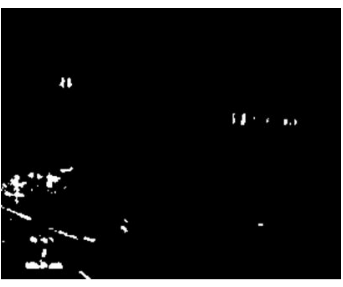

(b) Black top-hat transformation

Figure 6. Features based on black top-hat transform.

\subsection{Classifier Training}

All objects extracted from the segmentation stage were characterised by the features described in the previous sub-section. These features are eccentricity, circularity, ellipticity, rectangularity, triangularity, area, $x$-coordinate, $y$-coordinate, black top-hat value, maximum intensity (Max In), mean intensity (Mean In) and minimum intensity (Min In).

The extracted objects were classified by support vector machines (SVM) with a Gaussian radial bases function (RBF) kernel. The SVM was trained with 503 objects which were extracted for this purpose. The data set contains objects which represent headlight and non-headlight classes. A 10-fold cross-validation was chosen to train and test the classifier.

The classifier was trained with different combinations of the 12 features mentioned above in order to investigate which features have a significant effect on the classification and thus find the set of minimum number of features which minimises the classification error. This process was carried out in three experiments which are described as follows:

Experiment 1. In this experiment the SVM was trained with each of the 12 features separately. The training and testing was achieved using 503 objects with 10 -fold cross validation and the classification rate was computed for each combination. Table 3 illustrates the average classification rate of 10 -fold cross validation for each feature combination.

Experiment 2. To understand the effect of different feature combinations, features of the 503 objects were added one by one to train and test the SVM classifier. The average classification rate of the 10-fold cross validation computed for each combination is given in Table 4. 


\begin{tabular}{|c|c|c|c|c|c|c|c|c|c|c|c|c|c|}
\hline \multirow{3}{*}{$\begin{array}{l}\text { Feature } \\
\text { Combi- } \\
\text { nation }\end{array}$} & \multicolumn{12}{|c|}{ Features } & \multirow{3}{*}{$\begin{array}{l}\text { Classi- } \\
\text { fication } \\
\%\end{array}$} \\
\hline & \multirow{2}{*}{ Ecc } & \multirow{2}{*}{$\mathrm{C}$} & \multirow{2}{*}{$\mathrm{E}$} & \multirow{2}{*}{$\mathrm{R}$} & \multirow{2}{*}{$\mathrm{T}$} & \multirow{2}{*}{ Area } & \multicolumn{2}{|c|}{ Position } & \multirow{2}{*}{$\begin{array}{l}\text { Top } \\
\text { Hat }\end{array}$} & \multirow{2}{*}{$\begin{array}{c}\text { Max } \\
\text { In }\end{array}$} & \multirow{2}{*}{$\begin{array}{c}\text { Mean } \\
\text { In }\end{array}$} & \multirow{2}{*}{$\begin{array}{c}\text { Min } \\
\text { In }\end{array}$} & \\
\hline & & & & & & & $X$ & $\mathrm{Y}$ & & & & & \\
\hline $\mathrm{C} 1$ & $\checkmark$ & & & & & & & & & & & & 75.9 \\
\hline $\mathrm{C} 2$ & & $\sqrt{ }$ & & & & & & & & & & & 72.8 \\
\hline $\mathrm{C} 3$ & & & $\checkmark$ & & & & & & & & & & 73.6 \\
\hline $\mathrm{C} 4$ & & & & $\checkmark$ & & & & & & & & & 72.1 \\
\hline $\mathrm{C} 5$ & & & & & $\checkmark$ & & & & & & & & 73.8 \\
\hline C6 & & & & & & $\checkmark$ & & & & & & & 69.5 \\
\hline C7 & & & & & & & $\checkmark$ & & & & & & 68.6 \\
\hline $\mathrm{C} 8$ & & & & & & & & $\checkmark$ & & & & & 75.5 \\
\hline $\mathrm{C} 9$ & & & & & & & & & $\checkmark$ & & & & 68.8 \\
\hline $\mathrm{C} 10$ & & & & & & & & & & $\checkmark$ & & & 68.6 \\
\hline $\mathrm{C} 11$ & & & & & & & & & & & $\checkmark$ & & 68.3 \\
\hline $\mathrm{C} 12$ & & & & & & & & & & & & $\checkmark$ & 68.5 \\
\hline
\end{tabular}

Table 3. Classification with single feature.

Experiment 3. In this experiment, training and testing were carried out by eliminating one or two features from the 12 features in order to generate different combinations. The classification rate corresponding to each feature combination is given in Table 5 .

From the results of these three experiments it can be shown that area, triangularity, rectangularity, hat value and position are the most significant features while ellipticity, maximum and minimum intensity features are least significant. Therefore, features combination $\mathrm{C} 44$ was selected to be the one which was invoked to classify vehicle headlights.

\subsection{The Pairing Algorithm}

At night, features are almost reduced to pairs of spots representing headlights. These pairs can be employed to distinguish vehicles from other objects in the scene. A pairing algorithm designed to pair the two headlights can also be invoked to differentiate among other vehicles. In addition, it can be exploited to discard all other objects which pass the classification test because of their similarity to headlights. Therefore, the pairing process provides a way to improve the overall performance of the proposed system. 


\begin{tabular}{|c|c|c|c|c|c|c|c|c|c|c|c|c|c|}
\hline \multirow{3}{*}{$\begin{array}{l}\text { Feature } \\
\text { Combi- } \\
\text { nation }\end{array}$} & \multicolumn{12}{|c|}{ Features } & \multirow{3}{*}{$\begin{array}{l}\text { Classi- } \\
\text { fication } \\
\%\end{array}$} \\
\hline & \multirow{2}{*}{ Ecc } & \multirow{2}{*}{ C } & \multirow{2}{*}{ E } & \multirow{2}{*}{$\mathrm{R}$} & \multirow{2}{*}{$\mathrm{T}$} & \multirow{2}{*}{ Area } & \multicolumn{2}{|c|}{ Position } & \multirow{2}{*}{$\begin{array}{l}\text { Top } \\
\text { Hat }\end{array}$} & \multirow{2}{*}{$\underset{\text { Mn }}{\operatorname{Max}}$} & \multirow{2}{*}{$\begin{array}{c}\text { Mean } \\
\text { In }\end{array}$} & \multirow{2}{*}{$\begin{array}{l}\text { Min } \\
\text { In }\end{array}$} & \\
\hline & & & & & & & $X$ & $\mathrm{Y}$ & & & & & \\
\hline $\mathrm{C} 1$ & $\checkmark$ & & & & & & & & & & & & 75.9 \\
\hline C13 & $\checkmark$ & $\checkmark$ & & & & & & & & & & & 79.6 \\
\hline C14 & $\checkmark$ & $\checkmark$ & $\checkmark$ & & & & & & & & & & 84.1 \\
\hline C15 & $\checkmark$ & $\checkmark$ & $\checkmark$ & $\checkmark$ & & & & & & & & & 85.5 \\
\hline C16 & $\checkmark$ & $\checkmark$ & $\checkmark$ & $\checkmark$ & $\checkmark$ & & & & & & & & 85.9 \\
\hline C17 & $\checkmark$ & $\checkmark$ & $\checkmark$ & $\checkmark$ & $\checkmark$ & $\checkmark$ & & & & & & & 84.8 \\
\hline C18 & $\checkmark$ & $\checkmark$ & $\checkmark$ & $\checkmark$ & $\checkmark$ & $\checkmark$ & $\checkmark$ & & & & & & 87.4 \\
\hline C19 & $\checkmark$ & $\checkmark$ & $\checkmark$ & $\checkmark$ & $\checkmark$ & $\checkmark$ & $\checkmark$ & $\checkmark$ & & & & & 90.4 \\
\hline C20 & $\checkmark$ & $\checkmark$ & $\checkmark$ & $\checkmark$ & $\checkmark$ & $\checkmark$ & $\checkmark$ & $\checkmark$ & $\checkmark$ & & & & 91.9 \\
\hline C21 & $\checkmark$ & $\checkmark$ & $\checkmark$ & $\checkmark$ & $\checkmark$ & $\checkmark$ & $\checkmark$ & $\checkmark$ & $\checkmark$ & $\checkmark$ & & & 91.9 \\
\hline C22 & $\checkmark$ & $\checkmark$ & $\checkmark$ & $\checkmark$ & $\checkmark$ & $\checkmark$ & $\checkmark$ & $\checkmark$ & $\checkmark$ & $\checkmark$ & $\checkmark$ & & 92.1 \\
\hline $\mathrm{C} 23$ & $\checkmark$ & $\checkmark$ & $\checkmark$ & $\checkmark$ & $\checkmark$ & $\checkmark$ & $\checkmark$ & $\checkmark$ & $\checkmark$ & $\checkmark$ & $\checkmark$ & $\checkmark$ & 92.2 \\
\hline C33 & & $\checkmark$ & $\checkmark$ & $\checkmark$ & $\checkmark$ & $\checkmark$ & $\checkmark$ & $\checkmark$ & $\checkmark$ & $\checkmark$ & $\checkmark$ & $\checkmark$ & 91.7 \\
\hline $\mathrm{C} 32$ & & & $\checkmark$ & $\checkmark$ & $\checkmark$ & $\checkmark$ & $\checkmark$ & $\checkmark$ & $\checkmark$ & $\checkmark$ & $\checkmark$ & $\checkmark$ & 91.1 \\
\hline $\mathrm{C} 31$ & & & & $\checkmark$ & $\checkmark$ & $\checkmark$ & $\checkmark$ & $\checkmark$ & $\checkmark$ & $\checkmark$ & $\checkmark$ & $\checkmark$ & 90.5 \\
\hline C30 & & & & & $\checkmark$ & $\checkmark$ & $\checkmark$ & $\checkmark$ & $\checkmark$ & $\checkmark$ & $\checkmark$ & $\checkmark$ & 88.4 \\
\hline C29 & & & & & & $\checkmark$ & $\checkmark$ & $\checkmark$ & $\checkmark$ & $\checkmark$ & $\checkmark$ & $\checkmark$ & 85.1 \\
\hline C28 & & & & & & & $\checkmark$ & $\checkmark$ & $\checkmark$ & $\checkmark$ & $\checkmark$ & $\checkmark$ & 82.4 \\
\hline C27 & & & & & & & & $\checkmark$ & $\checkmark$ & $\checkmark$ & $\checkmark$ & $\checkmark$ & 81.6 \\
\hline C26 & & & & & & & & & $\checkmark$ & $\checkmark$ & $\checkmark$ & $\checkmark$ & 69.7 \\
\hline $\mathrm{C} 25$ & & & & & & & & & & $\checkmark$ & $\checkmark$ & $\checkmark$ & 68.8 \\
\hline $\mathrm{C} 24$ & & & & & & & & & & & $\checkmark$ & $\checkmark$ & 68.3 \\
\hline $\mathrm{C} 12$ & & & & & & & & & & & & $\checkmark$ & 68.5 \\
\hline
\end{tabular}

Table 4. Classification with different feature combinations.

Given an image with $n$ objects, the number of possible pairs $N_{p}$ can be given by

$$
N_{p}=\sum_{i=1}^{n-1} i .
$$

An image with 50 light spots contains 1225 possible pairs. Among them there are only a few pairs which may represent vehicles. This clearly explains the need to reduce the search space among the objects which facilitates finding the vehicles in the scene. It is achieved by discarding all the pairs which do not lie within the constraints specified by the pairing algorithm. 


\begin{tabular}{|c|c|c|c|c|c|c|c|c|c|c|c|c|c|c|}
\hline \multirow{2}{*}{$\begin{array}{l}\text { Feature } \\
\text { Combi- } \\
\text { nation }\end{array}$} & Ecc & $\mathrm{C}$ & $\mathrm{E}$ & $\mathrm{R}$ & $\mathrm{T}$ & Area & \multicolumn{10}{c|}{ Position } & Top & \multicolumn{1}{c|}{$\begin{array}{c}\text { Max } \\
\text { In }\end{array}$} & $\begin{array}{c}\text { Mean } \\
\text { In }\end{array}$ & $\begin{array}{c}\text { Min } \\
\text { In }\end{array}$ & $\begin{array}{l}\text { Classi- } \\
\text { fication }\end{array}$ \\
\hline C33 & & $\checkmark$ & $\checkmark$ & $\checkmark$ & $\checkmark$ & $\checkmark$ & $\checkmark$ & $\checkmark$ & $\checkmark$ & $\checkmark$ & $\checkmark$ & $\checkmark$ & 91.7 \\
\hline C34 & $\checkmark$ & & $\checkmark$ & $\checkmark$ & $\checkmark$ & $\checkmark$ & $\checkmark$ & $\checkmark$ & $\checkmark$ & $\checkmark$ & $\checkmark$ & $\checkmark$ & 91.3 \\
\hline C35 & $\checkmark$ & $\checkmark$ & & $\checkmark$ & $\checkmark$ & $\checkmark$ & $\checkmark$ & $\checkmark$ & $\checkmark$ & $\checkmark$ & $\checkmark$ & $\checkmark$ & 92.0 \\
\hline C36 & $\checkmark$ & $\checkmark$ & $\checkmark$ & & $\checkmark$ & $\checkmark$ & $\checkmark$ & $\checkmark$ & $\checkmark$ & $\checkmark$ & $\checkmark$ & $\checkmark$ & 91.2 \\
\hline C37 & $\checkmark$ & $\checkmark$ & $\checkmark$ & $\checkmark$ & & $\checkmark$ & $\checkmark$ & $\checkmark$ & $\checkmark$ & $\checkmark$ & $\checkmark$ & $\checkmark$ & 91.9 \\
\hline C38 & $\checkmark$ & $\checkmark$ & $\checkmark$ & $\checkmark$ & $\checkmark$ & & $\checkmark$ & $\checkmark$ & $\checkmark$ & $\checkmark$ & $\checkmark$ & $\checkmark$ & 91.2 \\
\hline C39 & $\checkmark$ & $\checkmark$ & $\checkmark$ & $\checkmark$ & $\checkmark$ & $\checkmark$ & & & $\checkmark$ & $\checkmark$ & $\checkmark$ & $\checkmark$ & 88.0 \\
\hline C40 & $\checkmark$ & $\checkmark$ & $\checkmark$ & $\checkmark$ & $\checkmark$ & $\checkmark$ & $\checkmark$ & $\checkmark$ & & $\checkmark$ & $\checkmark$ & $\checkmark$ & 90.5 \\
\hline C41 & $\checkmark$ & $\checkmark$ & $\checkmark$ & $\checkmark$ & $\checkmark$ & $\checkmark$ & $\checkmark$ & $\checkmark$ & $\checkmark$ & & $\checkmark$ & & 91.9 \\
\hline C21 & $\checkmark$ & $\checkmark$ & $\checkmark$ & $\checkmark$ & $\checkmark$ & $\checkmark$ & $\checkmark$ & $\checkmark$ & $\checkmark$ & $\checkmark$ & & & 91.9 \\
\hline C42 & $\checkmark$ & $\checkmark$ & $\checkmark$ & $\checkmark$ & $\checkmark$ & $\checkmark$ & $\checkmark$ & $\checkmark$ & $\checkmark$ & & & $\checkmark$ & 92.0 \\
\hline C43 & $\checkmark$ & $\checkmark$ & & $\checkmark$ & $\checkmark$ & $\checkmark$ & $\checkmark$ & $\checkmark$ & $\checkmark$ & & $\checkmark$ & & 91.8 \\
\hline C44 & $\checkmark$ & $\checkmark$ & & $\checkmark$ & $\checkmark$ & $\checkmark$ & $\checkmark$ & $\checkmark$ & $\checkmark$ & & & $\checkmark$ & 92.0 \\
\hline
\end{tabular}

Table 5. Classification rate of RBF classifiers with different feature combinations.

There are three parameters which play a central role in the design of the pairing algorithm

Distance between the two components in the image. Consider the pairing model illustrated in Figure 7. Let $d$ be the distance between the vehicle to be recognised and the camera, $f$ be the focal length of the camera, $d_{l}$ be the distance between the two headlights of the vehicle, and $d_{i}$ be the distance between the pair of the spots in the image.

Using triangle similarity, the distance between the two headlights is given by

$$
d_{i}=f \frac{d_{l}}{d} .
$$

Given a camera with a focal length of $200 \mathrm{~mm}$, for instance, the actual distance between the pair of headlights is $1.5 \mathrm{~m}$. When the vehicle is at a distance of $100 \mathrm{~m}$ from the camera, $d_{i}=3 \mathrm{~mm}$. When the vehicle is located at a distance of $10 \mathrm{~m}$ from the camera, $d_{i}$ becomes $30 \mathrm{~mm}$. Depending on the resolution of the imager, $d_{i}$ can be converted into pixels. Assuming that the imager has a resolution of 3.8 pixels/mm,

- the minimum horizontal distance between the two spots is 12 pixels,

- the maximum horizontal distance between the two spots is 114 pixels. 


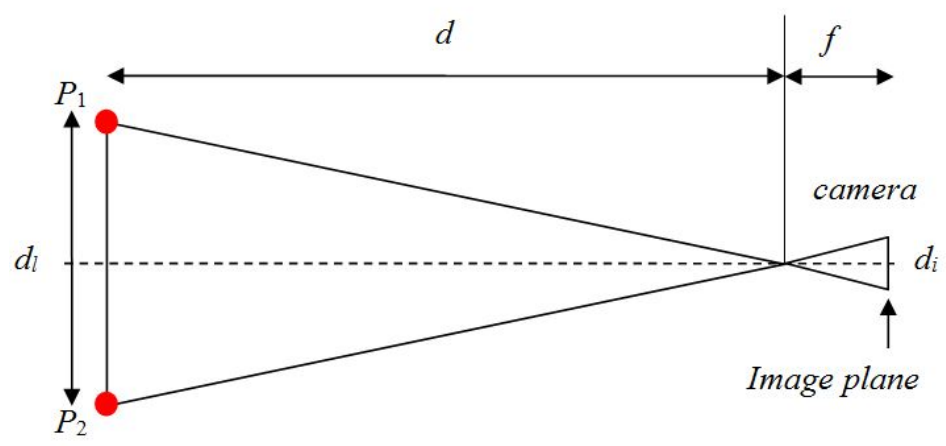

Figure 7. The pairing model.

Angle between the pair's two components. Due to the fact that headlights are almost horizontally aligned, the pairing algorithm should discard any pair whose two components create an angle with the horizontal direction more than a certain value. According to the measurements conducted on real-life images, this angle is specified as $\pm 5^{\circ}$.

Similarity of the pair's two components. One of the properties which can be very useful in the pairing algorithm is the similarity of the headlights. On the image, the two headlights generate similar spots with almost equal areas. For a moving vehicle towards the camera, the areas of these blobs are small when the vehicle is far away from the camera and their sizes increase as the vehicle under consideration approaches the camera.

\subsection{Pair Selection}

The proposed pairing algorithm is achieved by two stages:

Pair pre-selection. This algorithm starts by building an exhaustive list of all possible pairs in the image under consideration. A pairing rule tests all possible pairs for the validity of the following constraints:

- the horizontal distance between the two components,

- the angle between their centres,

- the ratio of the average area of the two components to the distance between them. 


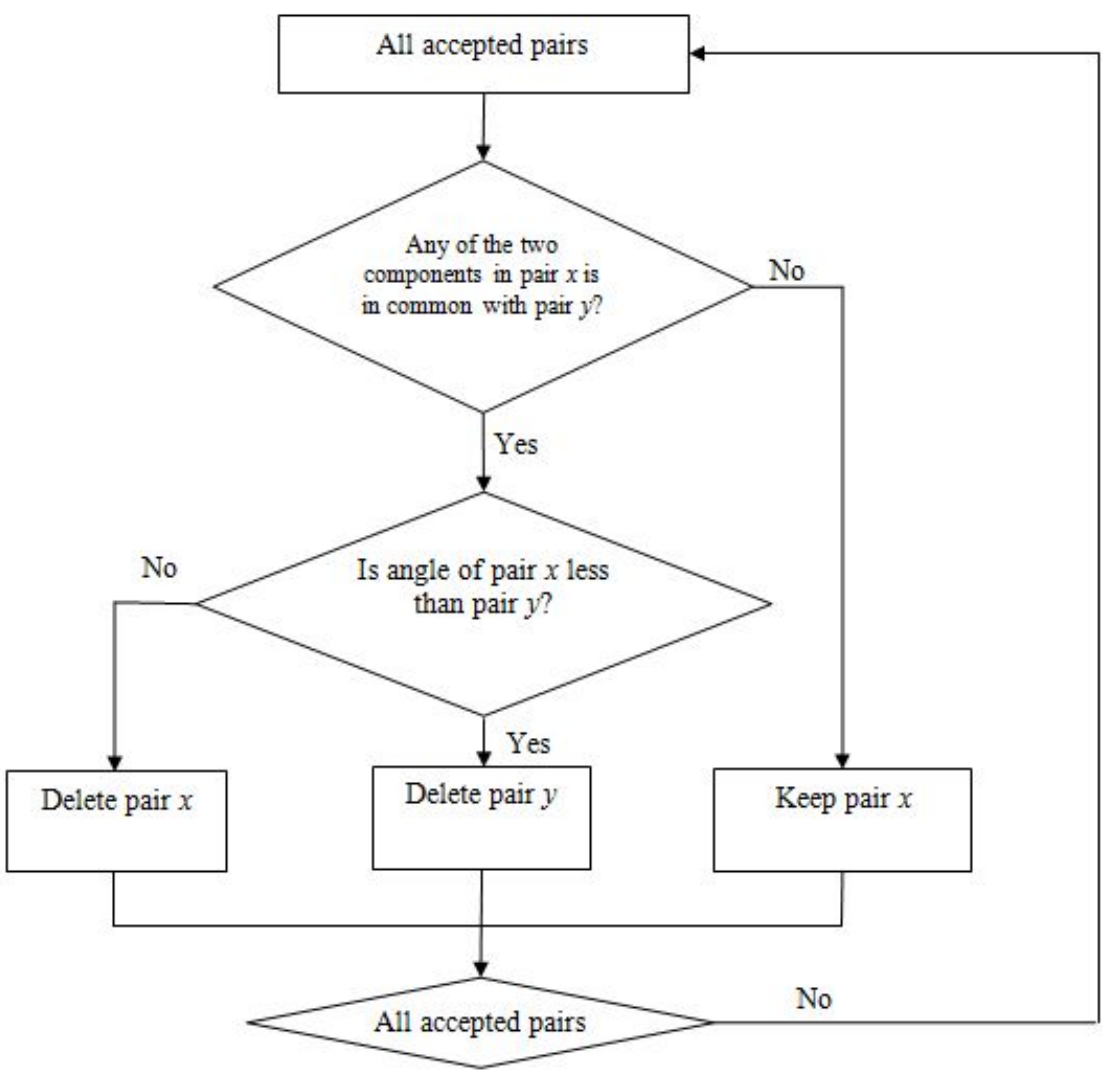

Figure 8. The pairing algorithm.

A pair is accepted only if all constraints are fulfilled by an AND operator. All pairs passing this rule are forwarded to a list of accepted pairs for further checking by the next stage.

Pair final selection. All pairs passing pre-selection are checked to identify whether one of their components pairs with another blob. If the two components do not pair with any other blob, the pair will be directly selected. On the other hand, if any of the two components pairs with any other blob, the angle between the new pair (denoted $y$ in Figure 8) and the horizontal line will be compared with that of the original pair (denoted $x$ in Figure 8). The pair with the smaller angle will be selected. 


\subsection{Multi-object Tracking}

Paired headlights are tracked by a set of Kalman filters. To achieve multi-object tracking, a set of discrete Kalman filters was invoked in an algorithm similar to that developed by Stauffer and Grimson [15]. The algorithm uses a number of Kalman filters to track the different objects simultaneously. In addition to the time and measurement updates of the Kalman filters, this algorithm has a data association part which is responsible for insuring an appropriate association between objects and Kalman filters and creates new Kalman filter trackers for new objects which appear in the image. Objects appearing anywhere in the image were given the same weight (same priority) since the pair of headlights could appear in the middle of the image.

\section{Results and Discussions}

The proposed vehicle detection algorithm was tested on 1410 frames which were collected from the 15 videos. There were 144587 objects extracted from the 1410 frames which were invoked to test the system. Of these objects, 3064 objects represented vehicle headlights. Figure 9 depicts the results of the different steps of the system and shows the vehicles which can be tracked by the system.

\subsection{Objects Classification}

Features of combination C44 were computed for all objects extracted from the videos invoked to test the proposed system. All of the 144587 objects were classified by the SVM classifier as headlight or non-headlight, where 3064 of them were extracted from headlights and 141523 from non-headlight classes. The performance achieved by the classifier is illustrated in Table 6 and the ROC graph in Figure 10. The classification rate achieved was $97.9 \%$ and the false positive rate was $2.4 \%$.

\subsection{The Paring Algorithm}

The pairing algorithm was tested with the 6394 objects which were classified by the SVM classifier as headlights. The proposed algorithm paired 3000 objects and rejected 3394 objects. Among the paired and rejected objects, it missed 23 vehicle headlight pairs and paired 57 pairs which were not vehicles headlights. Based on this analysis the performance of this algorithm is $94.6 \%$. 

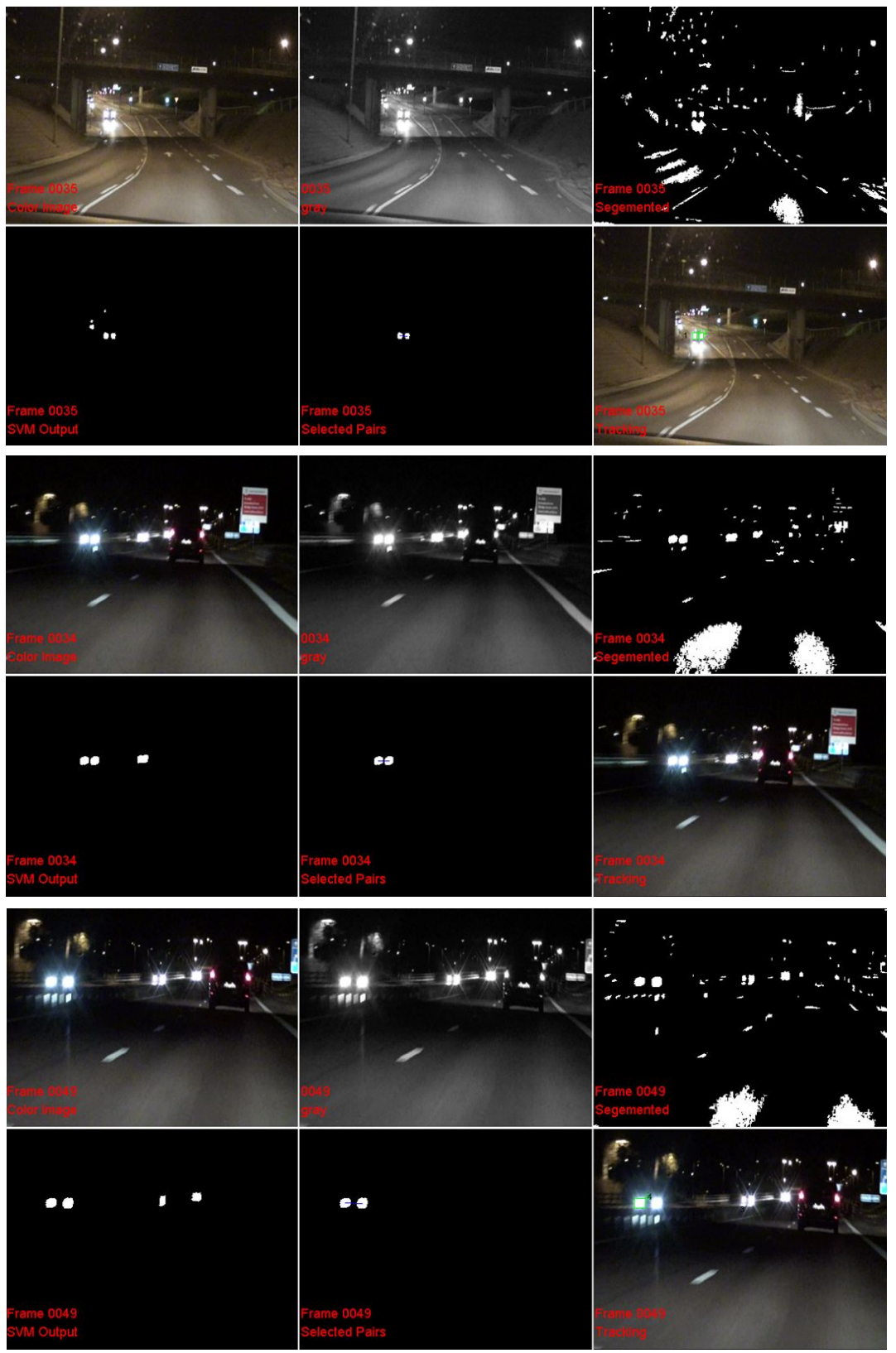

continued on next page 

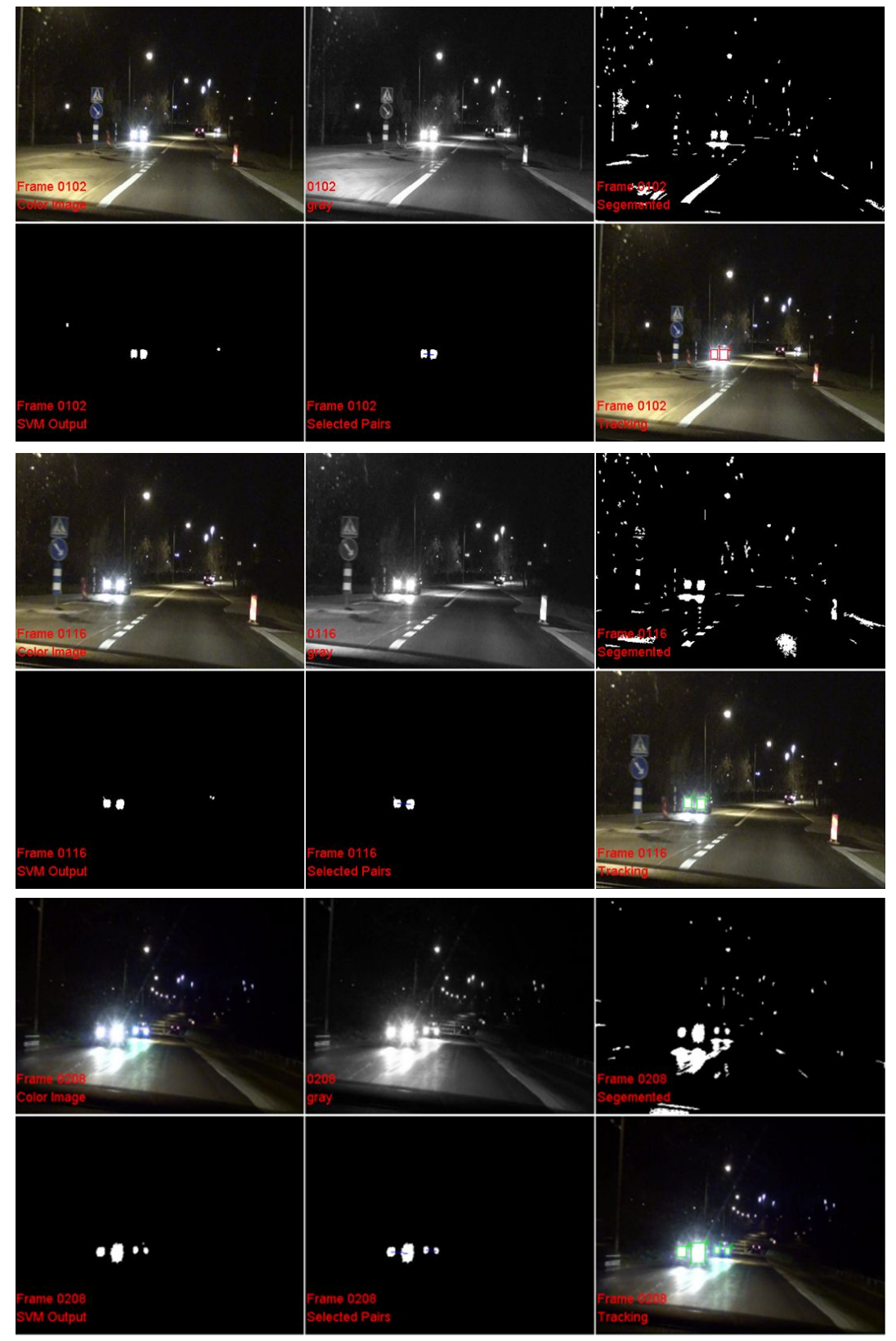

Figure 9. Sample results of multiple vehicle detection. 


\begin{tabular}{ccccc}
\hline Input & Total & \multicolumn{2}{c}{ SVM Output } & \multirow{2}{*}{ Error \% } \\
& & Headlight & Non-headlight & \\
\hline Headlight & 3064 & 3000 & 64 & 2.1 \\
Non-headlight & 141523 & 3394 & 138193 & 2.4 \\
\hline
\end{tabular}

Table 6. Error analysis of the single object classification achieved by the SVM classifier.

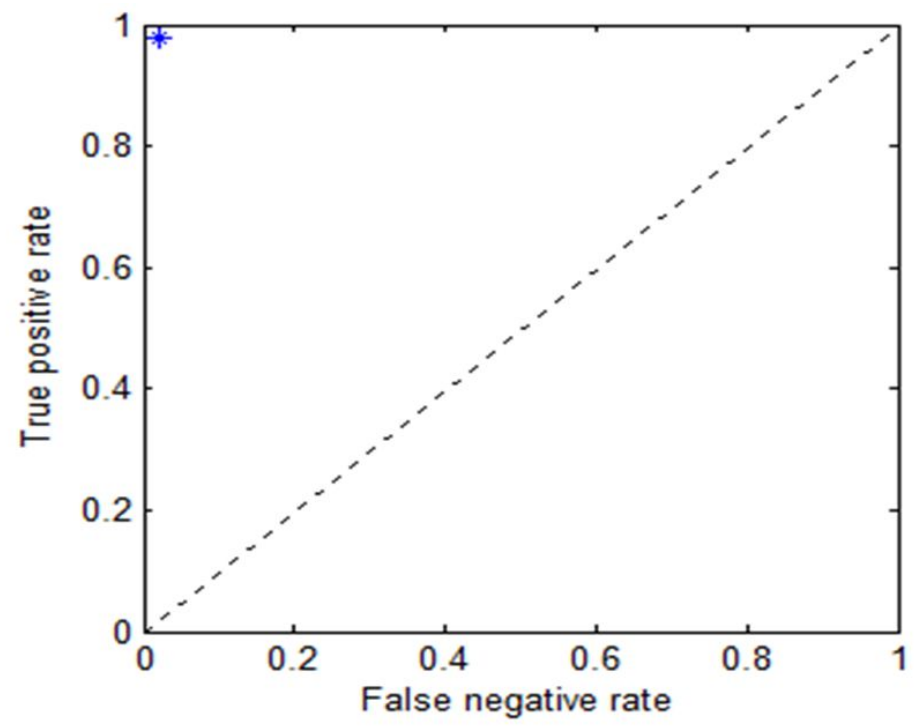

Figure 10. ROC graph showing the SVM performance.

\subsection{Testing the whole system}

The proposed approach was tested to detect and track 27 vehicles in night scenes. A successful case is considered when the vehicle was detected in the video and could be tracked by the tracker in a number of frames. Of the 27 vehicles, the system successfully detected and tracked 26 vehicles. Three vehicles were discarded from this test because in the first case the video was skewed, in the second case the vehicle was partially occluded by another vehicle, and in the third case the vehicle was on a curved road which cannot be tracked by the Kalman filter. The results of detection and tracking are given in Table 7. 


\begin{tabular}{ccc}
\hline & $\begin{array}{c}\text { Number of } \\
\text { detected and } \\
\text { tracked vehicles }\end{array}$ & $\begin{array}{c}\text { Detected and } \\
\text { tracked \% }\end{array}$ \\
\hline Detected and tracked & 26 & $96.3 \%$ \\
Failure & 1 & $3.7 \%$ \\
Total & 27 & \\
\hline
\end{tabular}

Table 7. Results of vehicle detection.

\subsection{Error Analysis}

In some cases, the tracking of the vehicle stopped for few frames and returned afterwards. An in-depth analysis showed that there are several reasons which can cause this problem. Vehicle merged headlights were responsible for $63 \%$ of the cases while $15 \%$ was caused by SVM misclassification of one or two headlights, $12 \%$ was caused by the pairing algorithm, and $10 \%$ caused by the tracking system. The problem of the merged headlights was due to glaring produced by the two headlights and the saturation of the pixels around them. This happened only when the vehicle was far away from the camera; as the vehicle approached the camera, this problem did not exist anymore. Figure 11 depicts the distribution of the error and gives reasons behind it.

The proposed approach was implemented using MATLAB and tested on a Hewlett-Packard Core2 Duo 2.00 GHZ processor and 4.00 GB RAM platform. The size of the image sequences employed for the test was $300 \times 200$ pixels. Two tests were conducted to evaluate the processing time. In the first test, the processing time of the algorithm for 1410 frames containing vehicles was measured. In this test 2558 frames were considered. These frames consisted of a mixture of frames containing vehicles and those without vehicles at all. The results of these two tests are depicted in Table 8 . The analysis indicates that more than $90 \%$ of the time is consumed by the feature extraction stage. This indicates the need to find other types of features which can reduce the processing time of this approach.

The proposed approach could successfully detect and track 26 out of a total of 27 vehicles in the videos under test. This represents a success rate of $96.3 \%$ of the cases used for the test. Comparison with previous research shows that the overall performance of the system proposed by Görmer et al. [8] was $89.3 \%$ and that proposed by Alt et al. [2] was $57 \%$ with high certainty, $16 \%$ with low certainty, and $27 \%$ for vehicle not detected. The system proposed by Liu et al. [10] could detect $99.4 \%$ of the vehicles in the videos, while the one proposed by Kim et al. 


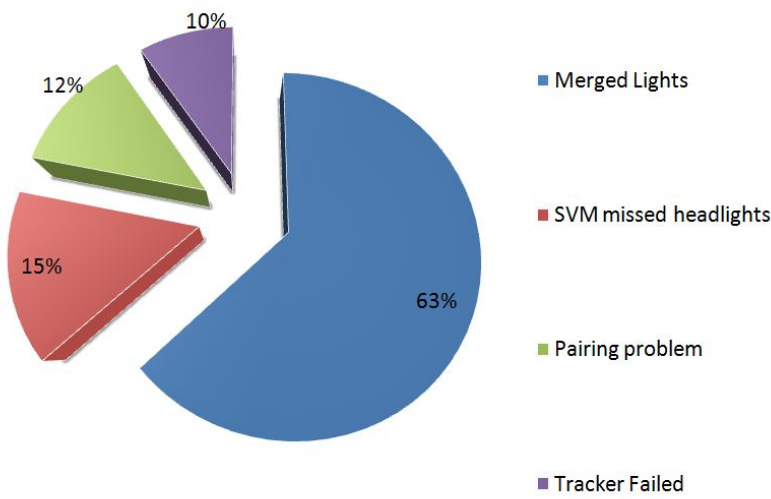

Figure 11. Failure analyses and error distribution.

\begin{tabular}{ccc}
\hline Task & $\begin{array}{c}\text { Test } 1 \\
\text { Time }(\mathrm{sec})\end{array}$ & $\begin{array}{c}\text { Test } 2 \\
\text { Time }(\mathrm{sec})\end{array}$ \\
\hline Colour conversion & 0.003 & 0.003 \\
Segmentation & 0.040 & 0.037 \\
Feature extraction & 0.926 & 0.820 \\
Classification & 0.020 & 0.017 \\
Pairing & 0.0001 & 0.0001 \\
Tracking & 0.010 & 0.007 \\
Total & 0.999 & 0.884 \\
\hline
\end{tabular}

Table 8. Average processing time of different tasks of the algorithm.

[9] achieved a successful rate of $85 \%$ for vehicle tracking. On the level of SVM classification, the current approach achieved $97.9 \%$ compared with $97 \%$ for the system proposed by Rebut et al. [12].

\section{Conclusions and Future Work}

This paper has presented an algorithm which is able to detect and track vehicles at night. The algorithm showed promising results as it detected and tracked 26 of 27 vehicles in the test population. A classification rate of $98 \%$ was achieved for the classifier which indicates that the features considered for the classification of 
headlights are suitable for this purpose. However, the time required to calculate these features should be minimised. The proposed algorithm was designed for vehicles with a pair of lights. Therefore, it cannot be used to detect vehicles with single light such as motorcycles.

A number of issues will be investigated in the future such as night vision conditions including rain or fog, exploring other segmentation techniques such as using colour temperature to segment the bright objects which could produce less unknown blobs, using other kinds of features which require less processing time, and using extended Kalman filters to enable tracking on curves.

\section{Bibliography}

[1] P. Alcantarilla, L. Bergasa, P. Jimenez, M. Sotelo, I. Parra, D. Fernandez and S. Mayoral, Night time vehicle detection for driving assistance lightbeam controller, in: IEEE Intelligent Vehicles Symposium (IV08), Eindhoven, Netherlands (2008), 291-296.

[2] N. Alt, C. Claus and W. Stechele, Hardware/software architecture of an algorithm for vision-based real-time vehicle detection in dark environments, in: Design Automation and Test in Europe (DATE '08), Munich, Germany (2008), 176-181.

[3] D. Bradley and G. Roth, Adaptive thresholding using the integral image, Journal of Graphics, GPU, \& Game Tools 12 (2007), 13-21.

[4] R. Cucchiara and M. Piccardi, Vehicle detection under day and night illumination, in: 3rd International ISC Symposium on Intelligent Industrial Automation and Soft Computing, Genova, Italy (1999), 1-4.

[5] A. Dubrovin, J. Leleve, A. Prevost, M. Canry, S. Cherfan, P. Lecoq, J. Kelada and A. Kemeny, Application of real-time lighting simulation for intelligent front-lighting studies, in: The Driving Simulation Conference, Paris, France (2000), 333-343.

[6] J. Flusser and T. Suk, Pattern recognition by affine moment invariants, Pattern Recognition 26 (1993), 167-174.

[7] J. Flusser, T. Suk and B. Zitová, Moments and Moment Invariants in Pattern Recognition, John Wiley \& Sons Ltd, Chichester, 2009.

[8] S. Görmer, D. Muller, S. Hold, M. Meuter and A. Kummert, Vehicle recognition and TTC estimation at night based on spotlight pairing, in: 12th International IEEE Conference Intelligent Transportation Systems, Louis, MO, USA (2009), 196-201.

[9] S. Kim, S. Oh, J. Kang, Y. Ryu, K. Kim, S. Park and K. Park, Front and rear vehicle detection and tracking in the day and night times using vision and sonar sensor fusion, in: IEEE/RSJ International Conference on Intelligent Robots and Systems, Alberta, Canada (2005), 2173-2178. 
[10] T. Liu, N. Zheng, L. Zhao and H. Cheng, Learning based symmetric features selection for vehicle detection, in: IEEE on Intelligent Vehicles Symposium (IV05), Las Vegas, NV, USA (2005), 124-129.

[11] N. Otsu, A threshold selection method from gray level histogram, IEEE Transactions on Systems, Man, and Cybernetics 9 (1979), 62-66.

[12] J. Rebut, B. Bradai, J. Moizard and A. Charpentier, A monocular vision based advanced lighting automation system for driving assistance, in: IEEE International Symposium on Industrial Electronics, Korea (2009), 311-316.

[13] P. Rosin, Measuring shape: ellipticity, rectangularity, and triangularity, Machine Vision and Applications 14 (2003), 172-184.

[14] P. Soille, Morphological Image Analysis: Principles and Applications, 2nd ed., Springer-Verlag, Berlin, 2010.

[15] C. Stauffer and W. Grimson, Learning patterns of activity using real-time tracking, IEEE Transactions on Pattern Analysis and Machine Intelligence 22 (2000), 747757.

[16] J. Sullivan, G. Adachi, M. Mefford and M. Flannagan, High-beam headlamp usage on unlighted rural roadways, Lighting Research and Technology 36 (2004), 59-67.

Received October 12, 2011.

\section{Author information}

Hasan Fleyeh, Computer E Department, School of Technology and Business Studies, Dalarna University, Rödavägen 3, 78188 Borlänge, Sweden.

E-mail: hfl@du.se

Iman A. Mohammed, Computer E Department, School of Technology and Business

Studies, Dalarna University, Rödavägen 3, 78188 Borlänge, Sweden.

E-mail: h09iamoh@du.se 